\section{Cahiers de Narratologie}

Analyse et théorie narratives

$31 \mid 2016$

Sérialité narrative. Enjeux esthétiques et économiques

\title{
Le problème de la sérialité narrative à la lumière du symbolisme des objets de consommation
}

\author{
Alessandro Leiduan
}

\section{(2) OpenEdition}

\section{Journals}

\section{Electronic version}

URL: http://journals.openedition.org/narratologie/7551

DOI: 10.4000/narratologie. 7551

ISSN: $1765-307 X$

Publisher

LIRCES

\section{Electronic reference}

Alessandro Leiduan, «Le problème de la sérialité narrative à la lumière du symbolisme des objets de consommation », Cahiers de Narratologie [Online], 31 | 2016, Online since 22 December 2016, connection on 20 April 2019. URL : http://journals.openedition.org/narratologie/7551; DOI : 10.4000/ narratologie.7551

This text was automatically generated on 20 April 2019.

Article L.111-1 du Code de la propriété intellectuelle. 


\title{
Le problème de la sérialité narrative à la lumière du symbolisme des objets de consommation
}

\author{
Alessandro Leiduan
}

Dans l'essai «L'innovation dans le sériel » (Eco 1985), Umberto Eco élève la sérialité au rang de paradigme esthétique de tout type de narration. Il n'y aurait de récit que sériel. Et si un récit nous semble " unique ", c'est que l'on méconnaît l'imbrication de son être avec celui des autres récits existants. Mais de quel type de "sérialité » parle Eco dans cet essai ? S'agit-il de la sérialité des romans policiers, des bandes dessinées ou des téléfilms? Pas seulement. La sérialité est, selon lui, reconnaissable à la présence, dans un objet déterminé, d'un ensemble de traits invariables et récurrents que l'objet en question partage avec d'autres objets du même type ${ }^{1}$ : la condition d'un récit sériel est donc celle d'une entité qui est imbriquée dans d'autres entités avec lesquelles elle forme un tout relativement homogène (la série proprement dite). Or, cette condition ne serait pas, selon Eco, l'affaire exclusive des récits paralittéraires, mais concernerait toutes sortes de récits, y compris ceux qui relèvent d'un domaine narratif généralement considéré comme « littéraire » ou « artistique » - un domaine, donc, sur lequel ne pèse aucun soupçon de compromission avec les lois du marché ou avec les contraintes de la production industrielle. Rien à voir avec une condition narrative qui serait le propre d'une époque dominée par l'industrialisation et la marchandisation des activités humaines. Une large partie de l'essai d'Eco est d'ailleurs consacrée à montrer que les exemples de sérialité sont omniprésents dans l'histoire de l'art, certains d'entre eux (les tragédies grecques ou les poèmes chevaleresques de la Renaissance) datant d'ailleurs d'une époque où la poiesis humaine se déployait encore à travers des formes qui ne pourraient en aucun cas être qualifiées $\mathrm{d}^{\prime}$ " industrielles ${ }^{2}$ ». Une telle notion de "sérialité », on l'aura compris, ne saurait en aucun cas être considérée comme une excroissance culturelle du taylorisme ou du fordisme. Ce type de "sérialité » se confond plutôt avec l'idée même de littérature, de musique, de peinture (ou, plus généralement, d'art) qui sert de référence à tous les métalangages critiques et esthétiques de la postmodernité : l'idée d'une entité (littéraire, musicale, picturale, etc.) que l'on ne peut explorer qu'en comparant, l'une à l'autre, les 
parties dont elle se compose, dès lors que chacune des parties en question (tel récit, telle composition musicale, telle œuvre d'art...) entretient forcément des relations avec les autres parties et que le tout qu'elles forment constitue un réseau de connexions, un «rhizome » (Deleuze et Guattari 1972), un "palimpseste» (Genette 1982), un système d'objets emboîtés. En parlant de «sérialité », Eco utiliserait-il donc, ici, une simple métaphore, sans que ce choix lexical implique la moindre confusion entre le domaine narratif et les domaines industriels et économiques?

2 En prenant le contrepied de ce point de vue, j'essaierai, dans le présent article, de démontrer que la redéfinition des critères qui président à l'appréciation (esthétique) et à l'analyse (critique) des œuvres narratives autour d'une pareille idée de "sérialité narrative » est une conséquence de l'industrialisation du mode de vie des sociétés occidentales modernes et, plus particulièrement, d'une transformation de notre sensibilité critique et esthétique provoquée par la manipulation d'objets de plus en plus standardisés, issus des chaînes de production sérielles de nos industries ${ }^{3}$. En postulant le caractère « sériel » de tous les récits, Eco n'aurait donc fait, selon moi, que rabattre l'être d'un objet symbolique comme le récit sur celui des autres objets matériels de type "sériel» qui servent de médiation instrumentale à toutes les actions que nous accomplissons au quotidien. Mais l'existence d'un tel lien de continuité entre les récits sériels et l'ensemble des produits sériels issus de l'univers de l'industrie fait l'objet, chez Eco, d'un refoulement idéologique. L'enjeu de son discours est d'accréditer l'existence d'une forme de «sérialité » transhistorique qui serait commune à toutes les époques de l'histoire (non uniquement à l'époque moderne) afin de «blanchir» les formes industrielles de "sérialité narrative " des soupçons de compromission avec les lois de l'économie qui pèsent généralement sur elles. C'est seulement en décryptant «à rebrousse-poil » l'argumentation développée par Eco dans l'essai «L'innovation dans le sériel » que l'on pourra en démasquer le caractère idéologique.

\section{Points de convergence entre récits sériels et objets sériels}

3 Il n'est meilleure façon, me semble-t-il, de vérifier cette hypothèse que d'appliquer à l'étude de l'interaction entre la société et les objets d'usage courant les mêmes catégories descriptives par lesquelles Eco essaie de rendre compte, dans l'essai «L'innovation dans le sériel », du mode de réception sociale des récits sériels. Si ces catégories parviennent à éclairer la nature de cette interaction, la thèse qui inspire cet article apparaîtra moins arbitraire qu'elle ne donne, peut-être, l'impression de l'être actuellement.

Tout texte présuppose, et construit toujours, un double lecteur modèle (disons un lecteur " naïf» et un lecteur "critique »). Le premier aborde l'œuvre en tant que dispositif sémantique, ce qui fait de lui une victime des stratégies de l'auteur, qui va le conduire petit à petit à travers une suite de prévisions et d'attentes; le second évalue l'œuvre en tant que produit esthétique et apprécie les stratégies mises en œuvre par le texte pour construire les compétences des Lecteurs Modèles de premier degré. Ce lecteur de second degré est celui qui aime la sérialité des séries, non pas tant à cause du retour d'un même contenu diégétique (que le lecteur naïf croyait différent) qu'à cause de la stratégie des variations; autrement dit, il apprécie la manière dont une même histoire (l'identico di base) est constamment retravaillée afin d'apparaître toujours différente (Eco $1985: 135, \mathrm{~m} . \mathrm{t}^{4}$.). 
Il n'est pas malaisé de comprendre ce qu'Eco veut dire dans ce passage à propos des modes de réception d'un récit sériel. Mais que pourrait vouloir dire ce même texte si on l'appliquait à l'interaction entre la société et les objets standardisés de l'industrie sérielle ? De quels types de conduites sociales les modèles idéal-typiques de lecteur dont Eco parle ici (lecteur de premier degré, lecteur de deuxième degré) seraient-ils les corrélatifs métaphoriques?

Pour le savoir, esquissons une sorte de phénoménologie du rapport que les sociétés actuelles entretiennent avec les objets sériels que le mode de production industriel met à leur disposition (par exemple des voitures, des vêtements, des meubles, des aliments, etc.) afin de nous doter d'un terme de comparaison qui nous servira, ensuite, à mesurer les points de convergence entre les récits sériels et les objets industriels.

\section{(i) Le déni de la fonction instrumentale}

7 Nous aurions tort de croire que le rapport que nous entretenons avec les objets de notre environnement quotidien est purement utilitaire. Certes, le statut de tous ces objets est celui d'un instrument : ils nous aident à accomplir la plupart de nos actions, mais ce n'est pas forcément pour venir à bout des objectifs qui devraient motiver l'accomplissent de telle ou telle autre action que nous les utilisons. Les voitures sont des moyens de transport, les meubles des moyens utiles à l'aménagement d'une habitation, les vêtements des moyens destinés à protéger le corps ; mais utilisons-nous toutes ces choses uniquement pour les finalités qui s'accordent le mieux aux fonctions que chacune d'entre elles est, en principe, apte à remplir ? Si tel était vraiment le cas, nous n'exigerions pas de disposer, à chaque fois que nous accomplissons une action impliquant une médiation instrumentale, d'un large éventail d'objets équivalents, susceptibles de remplir tous la même fonction et assurant des performances presque identiques (que l'on pense aux différents types de voitures présents sur le marché de l'automobile). De plus, parmi ces objets, nous ne convoiterions pas les plus couteux, mais notre choix se porterait systématiquement sur les moins chers, dès lors que les performances de ceux-ci seraient équivalentes à celles des autres objets. Or, comment se fait-il que les gens se comportent d'une tout autre façon et qu'ils convoitent, en général, les objets haut de gamme, n'achetant du bas de gamme que lorsqu'ils n'ont pas les moyens de s'offrir plus cher? C'est que l'usage de tous ces objets ne répond pas tant à la fonction qu'ils pourraient remplir en tant qu'entités matérielles qu'à la fonction qu'ils remplissent en tant qu'entités sémantiques. «Le vêtement, écrit Barthes, sert à se protéger, la nourriture sert à se nourrir, quand bien même ils servent aussi à signifier " (Barthes 1993 : 1478). La fonction sémantique se superpose à la fonction utilitaire et finit même, parfois, par l'écraser. La sémantisation d'un objet et, plus particulièrement, des finalités associées à son usage social, est inévitable : « Dès qu'il y a société, tout usage est converti en signe de cet usage » (Barthes 1993 : 1488). Mais, dans le cadre d'une société de consommation, le sens rattaché à l'usage d'un objet a ceci de spécifique qu'il est fort éloigné de l'utilité matérielle que pourrait avoir l'objet en question si on l'envisageait en tant que support d'un usage purement utilitaire. Les raisons qui poussent les consommateurs d'aujourd'hui à consommer certains vêtements, certains aliments ou certaines voitures, n'ont rien à voir avec les besoins que les objets en question seraient à même de satisfaire, s'ils étaient consommés pour leurs qualités matérielles. La publicité est le miroir où se reflète le plus clairement ce détournement de la fonction utilitaire des objets de consommation. Baudrillard soulignait de façon très pertinente que ce que l'on consomme vraiment, ce sont moins des objets que la publicité de ces objets (Baudrillard 1968: 205). Or, que nous 
disent généralement toutes les publicités sur les objets dont elles font la promotion ? Rares sont celles qui mettent en avant les avantages matériels que l'on pourrait escompter de l'utilisation de l'objet publicisé. Toutes ou presque investissent l'objet de significations très éloignées de celles qui pourraient rendre compte de la fonction que l'objet en question serait à même de remplir, si son usage social était indexé sur ses propriétés intrinsèques.

C'est donc parce qu'ils ne fonctionnent pas comme des instruments mais comme des entités sémantiques que les objets sériels peuvent être rapprochés des récits sériels. Mais quelle est la nature des significations qui servent à dissimuler la fonction instrumentale des objets sériels?

\section{(ii) La fonction affective des significations}

Les significations que possèdent les objets sériels n'ont pas vocation à décrire des états du monde, mais plutôt à exprimer l'état affectif d'un sujet à l'égard du monde. Ce sont des significations qui ne remplissent pas une fonction descriptive ${ }^{5}$, mais plutôt une fonction " expressive ", leurs effets sur la société se laissant caractériser en termes affectifs plutôt qu'en termes cognitifs. Décrypter ces significations revient donc à décrire les sentiments qu'elles mobilisent, à s'interroger sur l'origine de ces sentiments, sur les processus imaginaires qui président à leur surgissement. Les objets de consommation nous renseignent, en effet, sur la manière dont les sujets qui les achètent, les manipulent et, enfin, les détruisent, s'éprouvent eux-mêmes en tant qu'ils sont affectés par la représentation des objets en question. Si cette représentation interagit avec leur vie affective, c'est qu'elle confère à l'objet de consommation des propriétés que l'objet en question ne possède pas : des propriétés imaginaires, donc, qui n'ont rien à voir avec les propriétés réelles à l'aune desquelles les possibilités d'utilisation de tel ou tel autre objet devraient pouvoir être déterminées. L'impact affectif spécifique que l'on nomme « sentiment » n'est, en effet, autre chose que le résultat d'une interaction qui s'instaure entre un sujet et des entités, non pas réelles, mais imaginaires. Crédité par le discours publicitaire de propriétés qu'il ne possède pas, l'objet de consommation apparait alors aux consommateurs comme une sorte d'objet magique. En tant que tel, cet objet leur ouvre l'accès à des expériences qui sont considérées, malgré leur côté extravagant et, très souvent, irréalisable, comme constitutives du vécu d'une personne «normale ». Accéder à ces expériences - la remarque est fondamentale - n'est pourtant pas un loisir, une possibilité que les consommateurs d'aujourd'hui seraient en droit d'accepter ou de refuser, mais une obligation incontournable à laquelle il faut se plier sous peine de marginalisation sociale. Les publicités célèbrent ainsi le triomphe du conformisme, tout en donnant l'illusion d'être une incitation à la diversification des comportements sociaux, à l'utilisation la plus libre possible des objets sériels, à la transgression des codes de comportement dominants.

\section{(iii) une attitude sociale clivée entre naïveté et critique}

Cette phénoménologie du rapport que les sociétés actuelles entretiennent avec les objets sériels ne serait pas complète si elle faisait l'impasse sur la manière dont la société réagit aux formes sous lesquelles, par l'intermédiaire de ces publicités, les objets de consommation s'offrent à l'utilisation sociale. Il est évident que si la société était insensible aux boniments publicitaires par lesquels les objets de consommation cherchent à dissimuler leur nature "sérielle", en "se vendant" comme des facteurs de "personnalisation» des comportements humains, alors le modèle de société dit «de consommation» se serait effondré depuis longtemps. Or, comme ce modèle de société 
résiste encore, on pourrait penser que la société est complètement asservie à la logique qui permet à de simples objets utilitaires de fonctionner comme des objets symboliques. Mais n'y a-t-il vraiment personne aujourd'hui qui soit capable de s'apercevoir du caractère illusoire des expériences auxquelles les objets sériels nous promettent d'avoir accès? Non, la capitulation de la société occidentale actuelle aux formes symboliques « impures " par lesquelles les objets de consommation ont réussi à détrôner les valeurs (éthiques, politiques, religieuses, artistiques et philosophiques) autour desquelles gravitait autrefois la vie symbolique des sociétés traditionnelles n'est pas incompatible avec une prise de conscience plus ou moins aiguë du caractère illusoire, déviant et dénaturé de ce renversement symbolique. Nous ne nous rapportons pas à l'ensemble des biens de consommation que d'une manière naïve, mais aussi d'une manière critique. Il n'est probablement personne qui soit complètement aveugle au caractère fallacieux des promesses par lesquelles les objets de consommation flattent le narcissisme social en donnant l'impression à leurs utilisateurs d'être des moyens de "personnalisation " de leur identité sociale. Personne n'est donc complètement naïf, les attitudes concrètes de chacun d'entre nous oscillent entre un certain degré (jamais absolu) de naïveté et un certain degré (jamais nul) de méfiance critique.

Nous remarquons que, petit à petit, des convergences se font jour entre l'attitude de la société à l'égard des objets sériels d'usage courant et l'attitude qui caractériserait, selon Eco, la réception sociale des récits sériels. N'avait-il pas affirmé, dans le passage cité auparavant, que les récits sériels sont justiciables d'une double lecture : celle d'un lecteur naïf (ou lecteur de premier degré) et celle d'un lecteur critique (ou lecteur de deuxième degré)? Et n'avait-il pas caractérisé l'attitude de ces deux lecteurs en disant que l'un est " victime » des stratégies de séduction du récit, alors que l'autre est capable de décrypter ces mêmes stratégies (tout en étant incapable d'empêcher qu'elles produisent leurs effets) ? Lorsqu'on parle des objets et des récits «sériels", ne vise-t-on pas donc des entités qui interagissent avec la société à partir des significations qu'elles mobilisent ? La nature de ces significations n'est-elle pas, dans les deux cas, de nature affective?

Reste à savoir s'il s'agit de convergences vraiment significatives. Reste surtout à savoir si l'on peut vraiment rapprocher des choses aussi différentes que les récits et les objets d'usage au motif qu'elles participeraient toutes d'une certaine forme de « sérialité ».

\section{Le trait d'union « inavouable » entre récits sériels et objets sériels}

15 Reprenons certains passages du texte d'Eco que nous avons cité auparavant en les accompagnant, cette fois, d'un commentaire, dans le but de rendre encore plus légitime - si possible - l'hypothèse de lecture que nous sommes ici en train de développer. En caractérisant, tout au début du passage en question, l'attitude du lecteur de premier niveau, Eco affirme qu'il «aborde l'œuvre en tant que dispositif sémantique » (135). Arrêtons-nous, un instant, sur le terme «dispositif» (dispositivo) : il s'agit du mot par lequel on a traduit dans plusieurs langues (dont le français) le terme allemand de "Gestell», que le philosophe Heidegger utilise pour indiquer le pouvoir anonyme et impersonnel que la technique exerce sur l'homme. Un dispositif est donc quelque chose que l'homme a fabriqué mais qu'il ne contrôle plus et par lequel il se sent même contrôlé et dominé. L'usage de ce terme n'est sûrement pas fortuit, surtout si l'on admet l'hypothèse qu'ici, Eco pourrait parler, non pas seulement des récits sériels, mais aussi des 
autres objets sériels ainsi que des stratégies par lesquelles ces objets, à l'instar des récits, cherchent à contrôler, dominer et programmer le comportement de leurs utilisateurs. Parler de « dispositif» revient à dire que les entités en question (les récits et les objets sériels), bien qu'elles soient différentes, visent un même objectif: séduire la société, exercer sur ses comportements une forme de contrôle et de direction. Mais les similitudes ne s'arrêtent pas là car, à bien y regarder, la séduction des récits et des objets sériels opère à travers un même mécanisme: la mobilisation de significations affectives qui donnent l'illusion aux consommateurs piégés de se sentir vivre d'une vie qui n'est pas la leur. En considérant la nature de l'appât utilisé par les récits sériels, Eco parle d'un dispositif de type "sémantique " (dispositivo semantico: 135). Il pourrait dire la même chose - nous venons de le voir- des objets de consommation: eux aussi, par l'intermédiaire de la publicité, se vendent à la société comme des objets sémantiques, comme les supports d'un symbolisme, moins prestigieux peut-être, mais tout à fait équivalent à celui dont relèvent les récits. A la faveur d'une imprégnation sémantique de la conscience des consommateurs potentiels, les récits et les objets sériels font miroiter devant ceux-ci des simulacres identitaires qui leur donnent l'illusion de pouvoir transcender leur propre identité et de vivre des expériences qui semblent « coller » à leur personnalité authentique, mais qui n'en sont pas moins complètement "standardisées ». «Les prévisions et les attentes" (previsioni ed attese) à travers lesquelles se déploie le processus de séduction dont parle Eco ne concernent pas seulement, ici, le développement de l'intrigue d'un récit sériel, mais aussi, plus prosaïquement, les " scénarios imaginaires » que l'acheteur d'un bien attend qu'on lui offre et que le vendeur d'un bien prévoit qu'on lui demande. La destinée sociale d'un récit, tout comme celle d'un objet sériel, se réalise alors suivant les règles qui président au fonctionnement de cette institution "englobante " qu'est le marché : la nature d'un «objet sériel», qu'il soit immatériel comme un récit ou matériel comme une voiture, est celle d'un objet de consommation, c'est-à-dire d'un objet qui n'existe que pour être acheté, utilisé et vendu, le début et la fin de son existence coïncidant avec son entrée et sa sortie du marché. C'est parce qu'ils sont, à un même degré, les otages de ce mode d'existence « économique » que les récits et les objets sériels peuvent être considérés, malgré leurs différences, comme des excroissances d'une même notion de "sérialité ». Il est impossible pour eux de se dérober à cette condition, même s'ils venaient à interagir avec des destinataires capables de démonter, pièce après pièce, les mécanismes de séduction sur lesquels se fonde leur « appétibilité » commerciale. À en croire Eco, en effet, le lecteur critique (le «Lecteur Modèle de deuxième degré »), s'il n'est pas dupe des stratégies de séduction des récits sériels, c'est seulement parce qu'il s'accommode de la nature standardisée de ces récits et qu'il n'y cherche pas, contrairement au lecteur naïf, (le «Lecteur Modèle de premier degré »), un moyen d'entrer en symbiose avec les simulacres identitaires (les personnages) par lesquels ces récits essaient de "se vendre " pour ce qu'ils ne sont absolument pas: des facteurs de diversification des comportements sociaux. Le lecteur critique ne souffre pas du "retour de l'identique » parce qu'il sait que ce «retour » est inévitable dans un récit sériel: il circonscrit alors son bonheur de lecture à la reconnaissance des plus subtiles variations qui permettent à l'«identique " (la partie invariable de chaque épisode d'une série) de réapparaitre sous une apparence à peine dissimulée dans tous les récits de la même série.

Ce lecteur de second degré est celui qui aime la sérialité des séries, non pas tant à cause du retour d'un même contenu diégétique (que le lecteur naïf croyait différent) qu’à cause de la stratégie des variations; autrement dit, il apprécie la 
manière dont une même histoire (l'identico di base) est constamment retravaillée

afin d'apparaître toujours différente (Eco 1985 : 135, m.t.)

$\mathrm{Au} v \mathrm{vu}$ de ces remarques, il me semble qu'il y a bien lieu de poser un lien de continuité entre production sérielle de type artistique et industriel. Les catégories descriptives qui sont les plus aptes à caractériser l'interaction des sociétés actuelles avec ces deux types de productions sérielles sont à peu près les mêmes. La posture naïve ou critique que nous adoptons devant les récits existants (lorsqu'il s'agit de les apprécier esthétiquement ou de les analyser critiquement) est, en effet, calquée sur celle que nous adoptons face aux objets industriels de type sériel qui peuplent notre environnement quotidien et qui nous aident à accomplir la plupart de nos actions ou opérations. Certes, quand il parle de sérialité narrative dans l'essai dont il est question dans le présent article, Eco entend parler d'objet immatériels comme les récits, mais - la remarque est capitale - il ne dit pas des choses si différentes de celles qu'il dirait s'il parlait d'objets matériels et de la manière dont ils interagissent avec leurs utilisateurs. La raison en est que, dans un contexte où tout est voué à l'échange marchand, la forme sous laquelle les choses s'offrent désormais à l'attention humaine est la même, qu'il s'agisse de choses matérielles comme une voiture ou de choses immatérielles comme un récit.

\section{La sérialité « transhistorique » comme déguisement idéologique du symbolisme des objets de consommation}

Que penser alors de l'idée «transhistorique » de sérialité à travers laquelle Eco tente de repenser, dans cet essai, les formes « historiques » de sérialité pour les laver des soupçons d'une allégeance totale au marché ? Que penser du dialogisme, de l'intertextualité, de la littérature au second degré et des autres «avatars savants » de la sérialité narrative? Comment réussissent toutes ces notions (qui informent les pratiques de lecture officielles dans la quasi-totalité des milieux académiques) à passer pour l'expression d'un rapport " exemplaire " à la littérature narrative, exempt de tous les défauts qui discréditent les formes de réception courantes des séries diffusées par les mass medias? Pourquoi les interprètes qui cherchent à dévoiler les résonances intertextuelles, hypertextuelles, paratextuelles, transtextuelles, métatextuelles entre un récit et un autre n'ont pas l'impression de se faire complices, par ce type de lecture, d'une standardisation de la manière d'agir, d'être et de penser de la société ?

19 L'existence sociale de cette pratique culturelle à part que l'on nomme « récit » ne dépend pas seulement de l'existence d'objets (tel roman, tel film, tel livre de BD, etc.) en possession des caractéristiques formelles et thématiques que l'on observe généralement dans la plupart des récits existants. Elle dépend surtout de la manière dont tous ces objets 
interagissent avec la société. Sauf à vouloir faire coïncider les récits avec leur substrat matériel (manuscrit, volume illustré, bobine cinématographique, etc.), il faut bien reconnaitre que leur nature est celle d'un objet immatériel. Leur existence est donc redevable d'un acte mental : celui qu'il faut accomplir pour les produire et celui qu'il faut accomplir pour les interpréter. Or, la plupart des définitions que l'on trouvait, il y a encore quelques années, dans les manuels de narratologie, faisaient complètement abstraction de l'interaction qui s'instaure entre l'histoire racontée, d'un côté, et l'univers mental et affectif des producteurs et des destinataires d'un récit, de l'autre. Le récit était généralement défini comme une forme de communication qui articule (verbalement ou par d'autres moyens sémiotiques) un enchaînement d'actions ou d'événements. "La narration s'attache à des actions ou des événements » (Genette 1969: 59). Mais est-ce là l'essentiel ? Pas du tout. Le propre d'un acte de communication de type narratif (un récit) par rapport à un acte de communication de type non-narratif, c'est que son sens ne se laisse vraiment comprendre qu'à la faveur d'une "immersion" dans l'univers de l'histoire racontée (Schaeffer 1999): l'interprète doit revivre mentalement et affectivement les faits qui lui sont racontés. Si cette "immersion" psychoaffective de l'interprète dans l'univers de l'histoire racontée n'a pas lieu, le récit ne réalise pas pleinement les virtualités narratives qui sont inscrites dans son sens. Les virtualités narratives que tout récit porte en lui ne s'actualisent vraiment que lorsque le destinataire de ce récit parvient à capter les résonances affectives et mentales de l'histoire vécue par les personnages de l'histoire racontée, en envisageant cette histoire comme un prolongement de son expérience vécue. La force du récit, sa supériorité par rapport à toute autre forme de communication humaine, tient au fait que la compréhension de l'histoire racontée est à la mesure de la capacité de l'interprète de surmonter l'altérité de l'histoire en question, en reconnaissant un reflet de sa propre histoire personnelle dans les actes accomplis ou subis par le(s) protagoniste(s) de l'histoire lue ${ }^{6}$.

20 Faut-il conclure qu'il n'y a aucune différence entre les états psychiques et affectifs qui surgissent en nous pendant la lecture d'un récit et ceux qui émergent dans notre conscience au cours de nos interactions avec l'environnement social dans lequel se déroule notre existence réelle? Les «perceptions » sont-elles du même ordre que les « impressions » qui s'éveillent dans la conscience d'un lecteur de roman ou dans celle de quelqu'un qui regarde un film?

21 C'est la surestimation du risque d'une confusion identitaire entre le moi du lecteur et celui des personnages qui a autorisé les prises de position les plus absurdes à l'égard des genres narratifs, accusés de plonger les lecteurs dans un état psychique opaque, propice à la confusion de la réalité avec l'imaginaire (Lavocat 2016), propice donc à toutes sortes de manipulations idéologiques, publicitaires, politiques, etc. (le fameux storytelling). Cette incompréhension a favorisé aussi des prises de position, non moins absurdes, qui ont voulu restaurer la légitimité sociale des genres narratifs, en modifiant les critères qui président à leur réception sociale : les réflexes de lecture que les milieux académiques ont institutionnalisés au cours des dernières décennies (notamment grâce à l'initiative de certains « gourous » comme U. Eco) sont le résultat consternant d'une contre-éducation à l'appréciation esthétique et à l'analyse critique des œuvres narratives. La narratologie actuelle est, en partie, l'otage de ces réflexes de lecture « corrompus » : elle rend compte de comment les récits sont lus par des gens qui se sont méthodiquement appliqués à court-circuiter les réflexes de lecture "naturels » (sous prétexte qu'ils conviendraient à des lecteurs « naïfs » et non à des lecteurs « avertis »). 
Reconsidérons l'attitude du "lecteur naï » à l'égard de l'histoire racontée dans un récit (et, plus particulièrement, dans un récit sériel). Ce lecteur, nous dit Eco, ne s'aperçoit pas que l'histoire qu'il lit est la variation d'une autre histoire, il est insensible à la récursivité des situations autour desquelles cette histoire se développe et, malgré les nombreuses répétitions qui caractérisent l'intrigue des " séries ", il a toujours l'impression que chaque nouvel épisode de la série en question raconte une histoire qui est radicalement différente de celle qui a été racontée dans les autres épisodes. Il reconnaît une épiphanie de la différence, là où, paradoxalement, l'expressivité narrative atteint le comble de l'uniformisation (le «retour de l'identique»). S'il est victime de cette erreur d'appréciation, affirmerait Eco, c'est que le « lecteur naïf » considère l'histoire racontée comme un prolongement de son expérience vécue. Il ne se limite pas à déchiffrer son sens, il « revit » cette histoire (mentalement et affectivement), en cherchant en lui-même un écho des pensées et des sentiments des personnages. « Revivre » une histoire signifie, pour ce type de lecteur, l'envisager comme processus, non pas comme résultat. Une action est une action (ainsi pourrait-on caractériser la conception qui préside à sa compréhension de l'agir humain) non pas quand quelque chose arrive, mais quand il est possible de reconnaître dans ce qui est arrivé l'accomplissement d'une intention, c'est-àdire le passage à l'acte d'une volonté d'agir qui s'était manifestée auparavant à l'état virtuel. Saisir l'intention qui préside à l'agir d'une personne permet de prévisualiser le déroulement de l'ensemble de ces actes depuis le début jusqu'au résultat final. Avant même que l'action n'arrive à son terme, l'interprète est donc en mesure de prévoir comment le processus va se dérouler. «La séquence narrative n'est pas d'emblée une totalité réalisée, mais sa complétude est visée avant d'être atteinte » (Baroni 2007 : 92). Le problème de ce type de compréhension d'une histoire est, selon Eco, que l'interprète est fatalement happé par le déroulement de l'action racontée et qu'il ne peut pas l'analyser de l'extérieur, comme il le ferait s'il adoptait une perspective plus distanciée. Il perd alors conscience de toute médiation narrative et reçoit l'histoire racontée comme un prolongement de son expérience vécue. De ce fait, l'interprète est mis dans l'impossibilité de s'apercevoir que les actions racontées (et, à plus forte raison, les actions racontées dans un récit sériel) sont toutes hautement " prévisibles », conformes comme elles sont à une " casuistique » hyper-stéréotypée de l'agir humain. Le propre d'un récit sériel est de compenser la nature standardisée des actions racontées en usant d'un certain nombre de procédés narratifs (par exemple le cliffhanger) qui retardent l'accomplissement de l'action racontée, en empêchant ainsi le lecteur d'avoir une vision d'ensemble de l'histoire qu'on lui raconte. Mais que veut dire « retarder le déroulement de l'histoire racontée »? Toute histoire oscille entre des moments où les actions sont conçues à l'état virtuel (sous la forme de rêves, désirs, projets) et des moments où elles sont actualisées (conformément ou non aux intentions qui ont présidé à leur accomplissement). Or, le propre d'un récit sériel est de court-circuiter l'actualisation d'une action afin d'inscrire celle-ci dans un état de virtualisation prolongé, propice à des effets de tension narrative (Baroni 2007). De quelle manière ? (i) En cachant à l'interprète l'intention qui préside à l'agir d'un personnage (effet de curiosité) ; (ii) en le faisant douter de la capacité des personnages de venir à bout de leurs intentions (effet de suspense); (iii) en faisant aboutir l'action à un résultat différent de celui qui était visé par le personnage (effet de surprise). S'il n'arrive donc pas à se rendre compte que tous les processus racontés dans les histoires sérielles se déroulent autour d'un même schéma narratif, c'est que le lecteur naïf est, selon Eco, "prisonnier » de l'univers narratif où se déroule l'histoire qu'il doit interpréter. Et c'est cette condition de «captivité » (mentale et affective) qui court- 
circuite ses facultés critiques, en l'empêchant de reconnaître les similitudes qui existent entre l'histoire qu'il interprète et celles qui sont racontées dans les autres épisodes de la même série.

A l'opposé du "lecteur naïf », le «lecteur critique » envisage les actions racontées non pas comme des processus corrélés à une intention, mais comme des occurrences d'un certain type d'action. Il a une vision non pas dynamique mais statique de l'agir humain. Il ne prend pas en compte l'intention qui a présidé à l'accomplissement d'une action, mais la concordance entre l'action accomplie et les formes stéréotypées à travers lesquelles se déploie généralement l'agir humain ${ }^{7}$. Ce lecteur n'est pas prisonnier de l'histoire qu'on lui raconte, les actions dont elle se compose ne s'offrent pas à lui comme quelque chose d'unique, mais comme des occurrences d'un certain nombre de comportements codifiés qu'il a observés à maintes reprises dans le courant de sa vie. Les divers récits existants sont, à ses yeux, une vitrine des formes codifiées à travers lesquelles se déploient les comportements humains. La littérature narrative dans son ensemble est un "système de résonances » où chaque récit est l'« écho intertextuel» des autres récits. La sérialité devient l'horizon englobant de toute œuvre narrative, dès lors que les récits perdent leur autonomie, n'existant plus qu'en tant que pièces d'une mosaïque plus vaste, leur intelligibilité étant subordonnée à la compréhension des autres "épisodes » de cet ensemble (la série). Le présupposé de ce type de compréhension de l'agir narratif est une "casuistique générale de l'agir humain». J'appelle «casuistique» l'ensemble des constantes ontologiques du comportement humain en société, c'est-à-dire l'ensemble des régularités comportementales à partir desquelles l'agir de nos semblables nous devient intelligible. Pour donner un sens à une action, pour nommer cette action, pour soustraire les actes dont elle se compose à leur indétermination phénoménologique, il faut impérativement y reconnaître un reflet d'un certain modèle d'action répertorié dans notre conscience : c'est en ramenant à l'un de ces modèles toutes les actions observables au cours de notre vie que nous pouvons qualifier tel comportement de «vengeance », tel autre de «trahison », tel autre encore de " sacrifice », etc.

Quel est le modèle sémiotique qui préside à cette compréhension de l'agir humain? Le modèle sémiotique en question ne saurait être que celui qui rend compte de l'expressivité des objets sériels de consommation courante. Automobiles, meubles, lunettes, vêtements, parfums, boissons: tous ces objets donnent l'illusion à leurs utilisateurs de pouvoir déployer librement leur personnalité par-delà toute sorte de schéma de comportement codifié. Mais, en réalité, cette "personnalisation» de l'agir humain, cette "destandardisation des comportements sociaux » (Lipovetski 1993), n'est autre qu'un leurre : jamais les formes à travers lesquelles les êtres humains déploient leurs manières d'être, d'agir et de penser n'ont été aussi homogènes et régulières qu'elles le sont devenues depuis que le modèle de société dit «de consommation" s'est répandu à l'échelle planétaire (la mondialisation). Plutôt qu'exprimer la personnalité de leurs utilisateurs, les objets de consommation - devrait-on dire avec Baudrillard - «la désigne(nt) et la classe (nt)» (Baudrillard 1968: 228). Les préférences de consommation des membres d'une société désignent les individus en question en tant que membres d'une certaine classe dont la spécificité tient à des paramètres tels que l'âge, le sexe, le pouvoir d'achat que chaque individu partage avec des milliers (voire des millions) d'autres individus dans toute société avec un minimum d'étendue. Rien n'est donc moins expressif et " personnel» qu'une identité de ce type, du fait qu'elle assimile chaque consommateur individuel à quantité d'autres individus sur la base de caractéristiques, si communes et si 
générales, qu'elles ne rendent absolument pas justice (c'est le moins qu'on puisse dire) à ce qu'il peut y avoir d'unique dans le vécu d'une personne. Quel sens "personnel» pourrait, par exemple, être attribué à l'acte d'utiliser une automobile, à part celui qui a été attribué à cet objet par son fabricant (ou par les responsables de sa promotion commerciale)? Les seules variations personnelles semblent pouvoir relever, suggérait Barthes (1993), du style de conduite de l'automobile, mais il s'agit de "variations insignifiantes " qui n'infléchissent en rien la nature standardisée du sens inhérent à l'achat, à la possession et à l'usage de tel ou tel modèle de voiture. Il en va de même pour les autres objets de consommation : meubles, vêtements, gadgets. Aucun « fait individuel de novation » (Barthes 1993 : 1481) ne pourrait infléchir leur expressivité sémantique, car celle-ci se réduit à l'articulation d'un sens qui est inscrit dans ces objets mêmes avant leur utilisation (un sens que les usagers se limitent à actualiser conformément aux intentions des constructeurs et de leurs publicitaires). Dans le cadre de la sémiotique des objets de consommation, la Langue tient donc en otage la Parole : il ne peut pas y avoir d'actes de signification vraiment libres, la seule combinatoire possible mobilise des unités sémantiques prédéterminées selon des schémas fixés à l'avance ${ }^{8}$. Les seuls actes de signification possibles relèvent moins du registre expressif de la Parole que de celui de la Langue (il s'agit de «faits de Langue » déguisés en « faits de Parole»). Et c'est à ce type d'expressivité standardisée que des auteurs comme Umberto Eco ont conféré des lettres de noblesse en institutionnalisant dans les milieux érudits des réflexes de lecture qui sont (à l'insu de ceux qui pensent y reconnaitre l'expression d'une sensibilité culturelle supérieure) la transposition critique et esthétique des critères de réception à l'aune desquels la société de masse se rapporte à l'univers des objets sériels.

\section{Conclusion}

L'essai «L'innovation dans le sériel » est une tentative de dissimulation idéologique du processus à travers lequel se manifeste, dans le monde sérialisé contemporain, la soumission psychologique et affective de l'homme occidental à l'égard des objets de consommation. Le propre de l'idéologie n'est pas de dévoiler, mais de cacher les aspects négatifs du phénomène qu'elle entend légitimer (ici, le pouvoir régulateur que le marché exerce sur les comportements sociaux). Si ces aspects étaient rendus visibles, la société pourrait s'insurger contre l'ordre existant et dénoncer les mensonges qui permettent à cet ordre de s'auto-reproduire. Eco doit donc dissimuler la vraie nature de la sérialité narrative car, si on l'envisageait à partir de ses avatars les plus représentatifs (les récits paralittéraires diffusés par le réseau des mass media), elle révélerait une parenté embarrassante avec les objets sériels de la vie quotidienne. A cet effet, Eco s'est vu dans l'obligation d'anoblir le concept de « sérialité narrative », en montrant qu'il est applicable aussi aux formes narratives plébiscitées par les élites savantes. Envisagée ainsi, la « série narrative » devient un synonyme de dialogisme, d'intertextualité, de littérature au second degré et, plus généralement, de toutes les pratiques d'écriture fondées sur la citation, les guillemets, la parodie, etc.

Ce qui rend peu convaincante cette opération idéologique est l'ensemble des facteurs que nous avons dénoncés :

(i) L'attitude des lecteurs condamnés à être des victimes inévitables des stratégies de séduction de la narrativité sérielle est très proche de l'attitude de la société à l'égard des biens de consommation; 
28 (ii) La hiérarchie, établie artificiellement par Eco, entre lecteurs de premier et deuxième niveau, tend à disqualifier les réflexes de lecture les plus spécifiquement narratifs et à en institutionnaliser d'autres qui tendent à faire des récits non pas un « lieu d'interaction " entre le monde imaginaire et le monde de l'expérience mais, plus prosaïquement, la résultante d'une combinatoire d'un nombre fini d'unité thématiques et formelles.

Nous avons donc étudié, dans cet article, le phénomène de la sérialisation de l'imaginaire narratif ainsi que des critères qui président à son analyse critique et à son appréciation esthétique en tant que symptôme du processus d'appauvrissement de l'imaginaire social (et des pratiques symboliques auxquelles cet imaginaire était redevable de sa vitalité) sous l'effet du symbolisme des objets de consommation. La sérialité narrative est le symptôme de l'inféodation de l'imaginaire occidental à la logique qui préside au fonctionnement du symbolisme des objets de consommation. C'est pour compenser la sursignification des objets de consommation que la société a pu consentir à un appauvrissement des pratiques symboliques qui, à l'instar du récit, avaient traditionnellement vocation à fonctionner comme des termes de comparaison de l'expérience vécue, utiles à la compréhension, sur le mode de l'«exemplification modélisante " (Schaeffer 1999: 47), de l'agir humain. Les significations éthiques, politiques, philosophiques que l'on peut encore prêter aux œuvres narratives ne peuvent pas être réellement réinvesties dans le monde de l'expérience, car les réflexes de lecture qui gouvernent actuellement l'attitude de la société à l'égard des œuvres narratives ont tendance à brouiller toute perception, chez l'interprète, du lien de continuité existant entre son expérience vécue et l'expérience imaginaire représentée dans un récit. L'interprète est plutôt encouragé à vider les récits de tout ce qui pourrait encore permettre de reconnaître dans les histoires qu'ils racontent une sorte de prolongement imaginaire d'une "expérience vécue ". Vidé de tout pouvoir mimétique, le récit n'existe plus, dès lors, qu'en tant que vitrine des régularités formelles et thématiques qui caractérisent les autres récits existants. Chaque récit se rapporte à l'autre comme chaque épisode d'une série se rapporte à la série toute entière. La sérialité devient, de fait, une notion coextensive de l'ensemble de la littérature narrative. Croyant rompre avec des réflexes de lecture hérités de l'époque romantique qui assimilaient les œuvres d'art à quelque chose d'unique, d'original et de subjectif, les théoriciens qui, de la même façon qu'U. Eco, ont contribué à institutionnaliser ces nouveaux réflexes de lecture ont obéi, peut-être même à leur insu, à l'exigence de mettre en adéquation l'attitude de la société à l'égard des récits à celle qui caractérise l'attitude de cette même société à l'égard des objets de consommation. Les formes symboliques qui, autrefois, étaient dominantes (religion, éthique, philosophie, politique, art) sont devenues, désormais, subalternes au symbolisme impur des objets de consommation, obligeant ainsi la société à redéfinir à nouveaux frais les modalités de son interaction avec les genres symboliques qui, à l'instar des récits, ont encore vocation à stimuler l'imaginaire collectif. 


\section{BIBLIOGRAPHY}

Baroni, R. (2007), La tension narrative. Paris : Editions du Seuil.

Barthes, R. (1993), Euvres complètes. Paris : Editions du Seuil.

Baudrillard, J. (1968), Le système des objets. Paris : Denoël.

Benjamin, W. (2007 [1936]), L'œuvre d'art à l'époque de sa reproductibilité technique. Paris : Allia.

Deleuze, G. et Guattari, F. (1972), Capitalisme et Schizophrénie 1. L’Anti-Edipe. Paris : Minuit

Eco, U. (1985), Sugli specchi e altri saggi. Milano : Bompiani.

Genette, G. (1982), Palimpsestes. Paris : Editions du Seuil.

Genette, G. (1969), Figures II. Paris : Editions du Seuil.

Iser, W. (2012 [1969]), L'appel du texte. Paris : Allia.

Lipovetski, J. (1993), L'ère du vide. Paris : Gallimard.

Schaeffer, J.-M. (1999), Pourquoi la fiction? Paris : Editions du Seuil.

\section{NOTES}

1. «Du point de vue de la production industrielle en série, deux « occurrences » (tokens) peuvent être considérées comme des « répliques » du même «type » quand, en l'absence d'imperfections flagrantes, pour un individu normal (una persona normale), à partir d'un horizon d'attente normal (con esigenze normali), il sera indifférent de choisir l'une ou l'autre de ces deux « répliques ». Deux copies d'un film, deux exemplaires d'un livre, sont des répliques d'un même type " (Eco 1985 : 127). L'abréviation « m.t. » indique qu'il s'agit de ma traduction.

2. "Quand on considère la Poétique d'Aristote, on constate qu'il était possible de décrire le modèle de la tragédie grecque comme un modèle sériel. Les citations du Stagirite révèlent que les tragédies qu'il connaissait étaient bien plus nombreuses que celles qui sont parvenues jusqu'à nous, et qu'elles suivaient toutes (en le faisant varier) un même schéma fixe » (Eco 1985 : 144, m.t.).

3. En considérant le statut de l'œuvre d'art dans l'ère de sa reproductibilité technique, Walter Benjamin caractérisait dans les termes suivants l'impact du mode de vie industriel sur la sensibilité humaine: "Sortir de son halo l'objet en détruisant son aura, c'est la marque d'une perception dont « le sens de l'identique dans le monde » s'est aiguisé au point que, moyennant la reproduction, elle parvient à standardiser l'unique » (Benjamin 2007 [1936] : 21)

4. «Ogni testo presuppone e costruisce sempre un duplice Lettore Modello. Il primo usa l'opera come dispositivo semantico ed è vittima delle strategie dell'autore che lo conduce passo per passo lungo una serie di previsioni ed attese; l'altro valuta l'opera come prodotto estetico e valuta le strategie messe in opera dal testo per costruirlo appunto come Lettore Modello di primo livello. Questo lettore di secondo livello è colui che gode della serialità della serie e gode non tanto per il ritorno dell'identico (che il lettore ingenuo credeva diverso) ma per la strategia delle variazioni, ovvero per il modo in cui l'identico di base viene continuamente lavorato in modo da farlo apparire diverso » 
5. Et encore moins prescriptive, promissive ou déclarative, pour désigner les autres fonctions que les signes peuvent remplir, selon la terminologie de Searle.

6. Lire une histoire, c'est forcément lire l'histoire d'un autre. Mais, loin de rester prisonniers d'un sentiment d'altérité, la lecture d'une histoire nous permet de reconnaître un reflet de nous-mêmes dans le(s) protagoniste(s) de cette histoire. Nous avons alors l'impression, comme le dirait Iser, de contempler « moins les événements racontés que notre propre image dans un miroir » (Iser 2012 [1969] : 47). Les virtualités narratives d'un texte ne s'actualisent alors vraiment que lorsque nous arrivons à entrevoir l'image de notre visage sous les traits d'un être (réel ou imaginaire) que nous ne sommes pas.

7. Les formes stéréotypées de l'agir humain sont l'équivalent des unités narratives minimales dégagées par Barthes, dans $S / Z$, lorsqu'il définit les occurrences-types du code proaïrétique (telles que "promenade», "meurtre», "rendez-vous») sans guère se soucier des intentions qui président à l'agir des personnages du récit de Balzac.

8. En revanche, dans des systèmes sémiotiques moins sclérosés, le sens circule en deux directions : de la Langue à la Parole et de la Parole à la Langue. Les usagers de ces systèmes ont, certes, besoin de prélever leurs moyens d'expression dans le « trésor » de la Langue, mais celle-ci s'enrichit, à son tour, des effets de sens (G. Guillaume) les plus novateurs qui émanent des actes de signification concrets (la Parole) par lesquels les sujets expriment leur pensée personnelle. « Rien n'entre dans la langue qui n'ait été essayé par la parole » (Barthes : 1481). Cette double porosité rend compte de la vitalité d'un système sémiotique compatible avec des actes de signification non-standardisés.

\section{ABSTRACTS}

According to U. Eco, seriality is allegedly substantial to all narratives, not just the ones that have been designed to form a "series". This "serialization" of the narrative imagination, as well as of the criteria presiding over its critical analysis and its aesthetic appreciation is a result of the influence exercised by the symbolism of consumer goods on traditional symbolic practices. The purpose of this article is to show that the idea of literature, music, painting or, more generally, of art, which serves as a reference to all the critical and aesthetic metalanguages of postmodernity is only an ideological attempt to legitimate the forms through which the symbolic ascendancy of consumer goods displays its power on the behavior and the thought pattern of contemporary Western society.

Selon U. Eco, la sérialité serait le propre de tous les récits et non uniquement de ceux qui ont été conçus pour former une «série ». Cette "sérialisation» de l'imaginaire narratif ainsi que des critères qui président à son analyse critique et à son appréciation esthétique est une conséquence de l'emprise qu'exerce le symbolisme des objets de consommation sur les pratiques symboliques traditionnelles. L'objet de cet article est de montrer que l'idée de littérature, de musique, de peinture ou, plus généralement, d'art qui sert de référence à tous les métalangages critiques et esthétiques de la postmodernité, n'est qu'une tentative de légitimation idéologique des formes à travers lesquelles se déploie l'ascendant symbolique des objets de consommation sur la manière d'être, d'agir et de penser de la société occidentale contemporaine. 
INDEX

Chronological index: XXe siècle

Mots-clés: Eco Umberto, sérialité narrative, esthétique, société de consommation, symbolisme Keywords: Eco Umberto, narrative seriality, aesthetics, consumer society, symbolism Geographical index: Monde

\section{AUTHOR}

ALESSANDRO LEIDUAN

Université de Toulon, Babel, 2649

Alessandro Leiduan est maître de conférences à l'Université de Toulon. Ses recherches portent sur les relations entre pratiques symboliques et vie imaginaire. Il a dirigé plusieurs ouvrages collectifs, dont Entre fait et fiction (Modèles linguistiques 2012), Nouvelles frontières du récit. Au-delà de l'opposition entre factuel et fictionnel (Cahiers de narratologie $n^{\circ} 26,2014$ ). 